\section{Quinolone Antimicrobial Agents, 3rd Edition}

\section{David C. Hooper and Ethan Rubinstein}

\author{
American Society of \\ Microbiology Press, \\ Washington, DC 20036, USA \\ ISBN: 1-55581-231-7 \\ Pages: 485, Price: \$125.95
}

The quinolone class is one of the more important classes of antimicrobial agents discovered in recent years and one of the most widely used classes of antimicrobial drugs in clinical medicine. Their broad spectrum of activity and pharmacokinetic properties make the quinolone agents ideal for treating a variety of infections. Their clinical importance is further demonstrated by their activity against a wide range of diseases of public health importance such as anthrax, tuberculosis, bacterial pneumonia, and sexually transmitted diseases.

Recent research has provided new data on the agent's structure-function relationships, modes of action, resistance, pharmacokinetics, and drug interactions. The third edition of Quinolone Antimicrobial Agents nicely addresses these advances. The book is organized into four sections, each containing chapters written by leading experts.

Mechanisms and Spectrum of Activity and Resistance, the first major section, explores the basic biology of the quinolone class. The interrelationships between structure, antimicrobial activity, and side effects associated with various side chain positions of the quinolones are discussed here. Pertinent information on the bacterial topoisomerases and DNA gyrases, quinolone binding, DNA/RNA synthesis inhibition, cell death in the absence of protein synthe- sis, specific mutations within the quinolone resistance-determining region, and mutations that lead to altered access to target enzymes (efflux systems) is also highlighted.

In the next section, Pharmacology, the intricate field of quinolone pharmacokinetics (PK) and pharmacodynamics (PD) is evaluated. Information regarding absorption, distribution, metabolism, and excretion in a range of patient types is provided, followed by data gathered from pharmacokinetic/pharmacodynamic studies in a variety of models. This section also provides a review of the role of pharmacologic evaluation in optimizing therapy.

The last two sections of the book, Clinical Applications and Adverse and Other Effects, will be of particular interest to clinicians. The chapters in this section include a comprehensive reference on general considerations, antimicrobial aspects, treatment models, clinical and comparative studies, and suggested treatment regimens for a host of infections. The chapter on adverse effects has been greatly expanded from the second edition because a substantial body of new information has since been gathered. Topics covered here include allergic reactions, effects on connective tissue structures and pregnancy, phototoxicity, and central nervous system and immune system toxicity.

The organization and content of this text make it a superlative reference. The discussions on treatments and adverse effects contain some of the most current data available. The field of antimicrobial resistance, however, is one of the most rapidly evolving areas with constant discoveries of new cases and data. Users should understand that while this text serves as a valuable reference, other sources should be consulted to ensure that the most comprehensive data are obtained. The references provided throughout, however, give the reader starting points to other literature.

\section{Gregory J. Anderson*}

*Centers for Disease Control and Prevention, Atlanta, Georgia, USA

Address for correspondence: Gregory J. Anderson, Centers for Disease Control and Prevention, Mailstop C12, 1600 Clifton Road, Atlanta, GA 30333, USA; fax: 404-639-4197; email: gca5@cdc.gov

\section{Risk Analysis of Prion Diseases in Animals}

\section{David Adams and \\ Corrinne Ida Lasmézas, editors}

\section{OIE Scientific and Technical \\ Review 22 (2003) \\ ISBN 92-9044-573-4 \\ Pages: 346, Price: 45 Euros}

Although scrapie, a prion disease of sheep, has been recognized since the 18th century, it was the dramatic emergence of bovine spongiform encephalopathy (BSE) in British cattle in the late 1980s that brought the dangers of prion diseases into prominence. The subsequent spread of this disease into humans as new variant Creutzfeldt-Jakob disease (CJD) remains one of the unsolved emerging infectious disease mysteries of the 20th century. This issue (OIE Scientific and Technical Review 22 [2003]) of the Scientific and Technical Review of the Office International des Epizooties comes 11 years after a previous issue about BSE. Much has happened since then; this multi-author volume provides an excellent account of what is known about prion diseases in animals, including BSE, which has now spread from the United Kingdom to 14 other countries, and remains an important risk to human health. As a consequence of BSE emergence, research 
has been expanded considerably into transmissible spongiform encephalopathies (TSE). This research has resulted in improved diagnostic tests, which have contributed to risk management, even though our understanding of the underlying molecular pathogenesis of TSE remains limited.

The 17 chapters in this book are written by experts from many countries and illustrate the various approaches to risk management in specific regions of the world. In addition to BSE, the book contains chapters about TSE of North America such as scrapie, transmissible mink encephalopathy, and chronic wasting disease of deer and elk; the last disease has recently spread from its endemic Colorado-Wyoming area to six other states in the United States, as well as Alberta and Saskatchewan in Canada.

With the recognition that BSE had spread in the United Kingdom and many other countries through feeding contaminated mammalian meat-andbone meal to ruminants, a ban on this practice was instigated in the European Union in 1994. This ban has since been adopted by many other regions of the world. In 2000, the European Union strengthened the ban to prohibit feeding processed animal proteins to farmed animals kept, fattened, or bred to produce food. This measure has undoubtedly helped to prevent or reduce numbers of cases of BSE in ruminants but incidentally has led to a new science-based industry for feed analysis. This industry uses sophisticated molecular techniques such as near infrared spectroscopy and microscopy, polymerase chain reaction, and immunoassays to check feed for animal by-products, and to prevent intraspecies recycling (cannibalism), which undoubtedly caused the BSE epidemic that resulted in the death of 186,000 cattle from 1985 to 2002.

I recommend this book as an excellent source of information on all aspects of prion diseases in animals.

\section{Brian W.J. Mahy*}

${ }^{*}$ Centers for Disease Control and Prevention, Atlanta, GA, USA

Address for correspondence: Brian W.J. Mahy, Centers for Disease Control and Prevention, Mailstop C12, 1600 Clifton Road, Atlanta, GA 30333, USA; fax: 404-639-4197; email: bxm1@cdc.gov

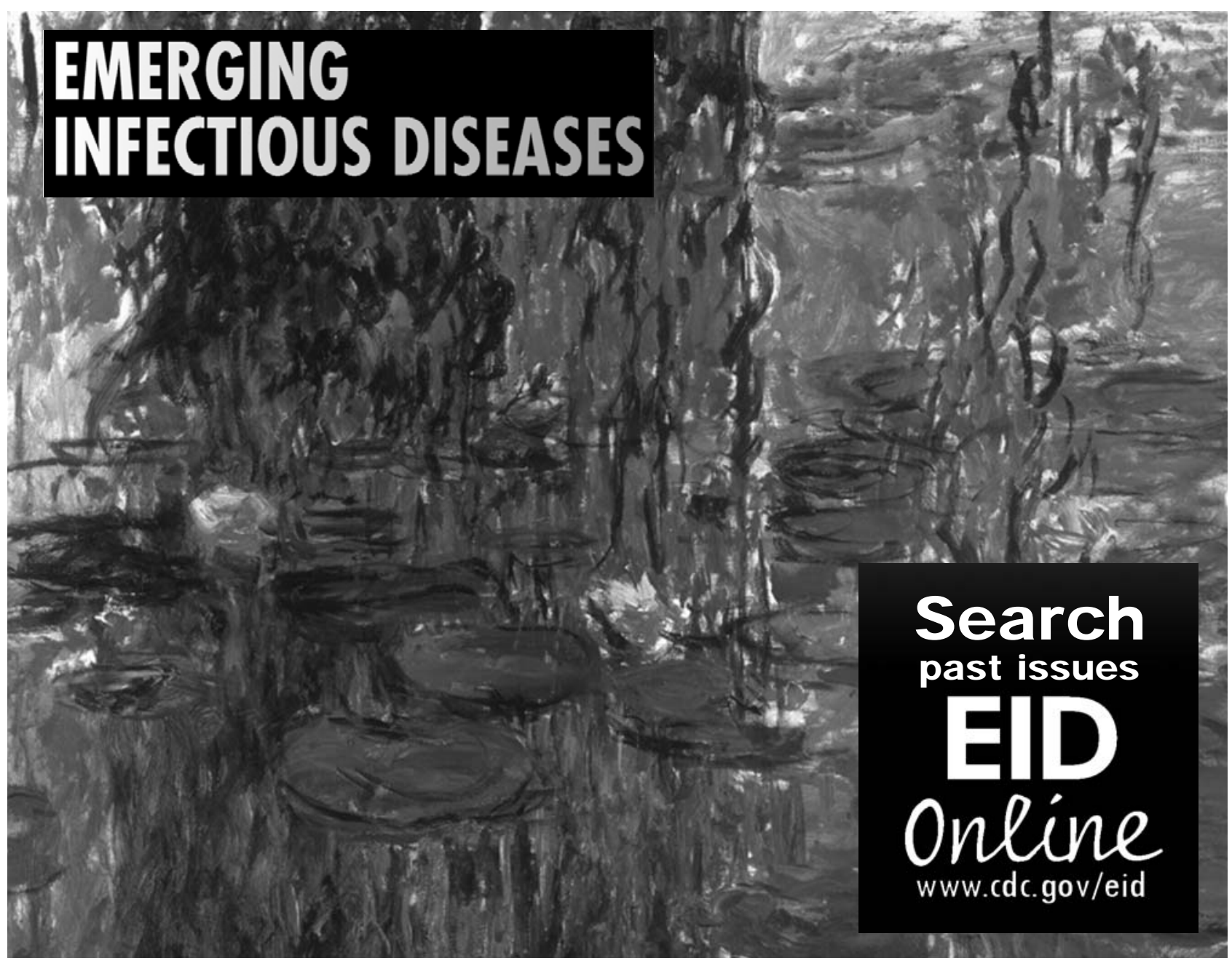

